\title{
Progress Toward a Km-Scale Neutrino Detector in the Deep Ocean
}

\section{MASTER}

R.G. Stokstad

\section{Nuclear Science Division}

November 1997

Presented at

Neutrinos in Astro,

Particle and Nuclear Pbysics,

Erice, Italy,

September 16-24, 1997, and to be published in

Progress in Particle and

Nuclear Pbysics, Vol. 40
RECEIVED JUN 021998

OSTI

DISTRIBUTION OF THIS DOCUMENT IS UMUMUTED 


\section{DISCLAIMER}

This document was prepared as an account of work sponsored by the United States Government. While this document is believed to contain correct information, neither the United States Government nor any agency thereof, nor The Regents of the University of California, nor any of their employees, makes any warranty, express or implied, or assumes any legal responsibility for the accuracy, completeness, or usefulness of any information, apparatus, product, or process disclosed, or represents that its use would not infringe privately owned rights. Reference herein to any specific commercial product, process, or service by its trade name, trademark, manufacturer, or otherwise, does not necessarily constitute or imply its endorsement, recommendation, or favoring by the United States Government or any agency thereof, or The Regents of the University of California. The views and opinions of authors expressed herein do not necessarily state or reflect those of the United States Government or any agency thereof, or The Regents of the University of California. 


\section{DISCLAIMER}

Portions of this document may be illegible electronic image products. Images are produced from the best available original document. 


\title{
Progress Toward a Km-Scale Neutrino Detector in the Deep Ocean
}

\author{
R.G. Stokstad \\ Institute for Nuclear and Particle Astrophysics \\ Nuclear Science Division \\ Emest Orlando Lawrence Berkeley National Laboratory \\ University of California \\ Berkeley, California 94720
}

.November 1997

This work was supported by the Director, Office of Energy Research, Office of High Energy Physics, Office of High Energy and Nuclear Physics, of the U.S. Department of Energy under Contract No. DE-AC03$76 \$ F 00098$. 


\title{
Progress Toward a Km-scale Neutrino Detector in the Deep Ocean
}

\author{
R. G. Stokstad \\ Institute for Nuclear and Particle Astrophysics \\ Lawrence Berkeley National Laboratory \\ Berkeley, CA 94720 USA
}

\begin{abstract}
The best particles for observing distant objects are photons and neutrinos. Because of the neutrino's weak interaction cross section, detectors suitable for astronomy must be very large and well shielded from cosmic rays. Eventually, a detector with the order of a square $\mathrm{km}$ of effective area will be needed for systematic observations of distant point sources such as active galactic nuclei. Prototype detectors are currently being developed at several sites in the ocean, at Lake Baikal, and in Antarctica. This talk summarizes the status of the projects that use the deep ocean for the detector medium and shielding: DUMAND, NESTOR and ANTARES. Technical developments will be needed for a future $\mathbf{k m}$-scale detector, progress on one of these, a digital electronic system, is also described.
\end{abstract}

\section{Introduction}

High energy neutrino astronomy is a scientific field in birth. It has been a long gestation period but, we believe, worth the wait. And the joyous event is near at hand - perhaps only a few years from now. Supernova 1987A (and the detection of its low-energy neutrinos by IMB and Kamiokande) was the harbinger, the star in the East announcing the coming of this new field. We will be sure this new scientific field has arrived when we are able to detect and identify high energy neutrinos having a galactic or extragalactic origin.

The science we hope to explore with a $\mathrm{km}$-scale neutrino detector is broad and potentially rich [1]. The basis for this statement, in part, is that the neutrino sky is still unexplored. (The photon sky and the cosmicray sky are by comparison well mapped.) With the exception of Supemova 1987A, noted above, neutrinos with an extra-solar-system origin have not yet been identified. Yet, the observation of high-energy cosmic rays guarantees there are neutrinos coming from regions of our galaxy and very likely from extra galactic sources as well. The clearest illustration of this is our observation of atmospheric neutrinos - neutrinos originating from the interactions of cosmic rays with the earth's atmosphere. By way of the same "beamdump" mechanism (generation of pions, whose decay leads to muon and electron neutrinos), high-energy cosmic rays in a galaxy will interact with distributed or concentrated matter to produce neutrinos of lower but comparable energy. From then on these neutrinos will travel undeflected by magnetic fields and virtually unabsorbed from their point of origin to us.

To be published in "Progress in Particle and Nuclear Physics" Vol.40. (Elsevier - Oxford). Proc. Int.School of Nuclear Physics, 19th Course:

"Neutrinos in Astro, Particle and Nuclear Physics" Erice, Sicily, September 16-24, 1997 
Neutrinos are thus an ideal tool for astronomy. Charged-particle trajectories are bent by galactic and intergalactic magnetic fields whose magnitudes are not well known. Even protons with energies as high as $10^{20} \mathrm{eV}$ may be deflected if their sources are sufficiently distant. Gamma-rays, while undeflected, are subject to absorption by intervening dust or at very high energies by interaction with the cosmic microwave background. In direct analogy to solar neutrinos, which enable us to study the core of the sun in real time, high-energy neutrinos should permit us to see the inner regions of quasars, active galactic nuclei, gammaray bursters and the panoply of cosmological point sources of high-energy particles. Neutrinos may confirm or even reveal the sources of the highest energy cosmic rays and enable the study of objects that would otherwise be invisible. The price, of course, is that neutrinos are difficult to detect. This, coupled with low fluxes, requires that the detectors be very large and well shielded. This led the pioneers of the field to propose a Cherenkov detector located deep in a large body of water. Theoretical predictions of fluxes suggest that the natural scale for a high energy neutrino telescope is a kilometer.

Rounding out the motivation for constructing a large neutrino telescope is a variety of experiments in nuclear and particle astrophysics that complement experiments or searches using other methods. Some of these topics - searches for WIMPS and magnetic monopoles, studies of atmospheric neutrinos and cosmic-ray composition, establishing supernovae and gamma-ray burst alarms - can be addressed by 'smaller' scale detectors $\left(0.1\right.$ or $\left.0.02 \mathrm{~km}^{2}\right)$ that are operating now or will be in the future. Finally, large arrays of sensors located in deep water (or even in ice) offer interesting possibilities for studies in the biological and earth sciences, including oceanography, seismology, and geology.

In this talk I will describe the progress being made toward a $\mathrm{km}$-scale neutrino detector in the ocean. While the scientific goals are largely independent of the medium (water or ice), the design of a detector and the logistics of building and operating it are sufficiently medium-dependent that a convenient, if scientifically arbitrary, classification arises. Thus, the detector located in polar ice, AMANDA, will be described in a talk by Francis Halzen; the one in deep fresh water, Lake Baikal, by Christian Spiering; and I will discuss the Pacific (DUMAND), the Mediterranean (NESTOR and ANTARES) and some recent developments at my own institution, LBNL. There is an additional contribution on ANTARES, by Francois Blondeau.

\section{DUMAND}

The DUMAND project (Deep Underwater Muon and Neutrino Detector) was the first of its kind [2]. Although the first documented suggestion of a large, underwater neutrino detector dates back to 1960 , it was not until the early 1970's that serious discussion began, leading eventually to a proposal in 1978 for R\&D [2]. A site $30 \mathrm{~km}$ off the Big Island of Hawaii was selected and work begun to characterize it, develop the hardware, and the techniques to deploy it. The basic concept developed by DUMAND for a prototype underwater neutrino detector is shown in Fig. 1. (DUMAND's conceptual array of 1978 was a much larger hexagonal structure, $800 \mathrm{~m}$ on a side.) The signature of a neutrino is a muon traveling upward from below the horizon, and the muon is observed and tracked using the Cherenkov radiation produced as it moves through the water. The photons are detected by large photomultiplier tubes (PMT), $38 \mathrm{~cm}$ in diameter, contained in glass pressure vessels mounted at intervals of (typically) $10 \mathrm{~m}$ on long mooring lines 
or "strings" spaced $40 \mathrm{~m}$ apart. The strings are connected electrically to a central junction box, which, at $4800 \mathrm{~m}$ depth, is the terminus for an electro-optical cable leading to the shore station, $30 \mathrm{~km}$ away. The cable provides electrical power for the PMT's and the electronics, and the optical fiber carries digitized information from each event satisfying a local trigger requirement.

Central to this concept is the ability to deploy each string at its intended location and then make electrical

\section{Dumand II. Octagonal Neutrino Telescope} Aray dimensions: $200 \mathrm{~m}$ high, $100 \mathrm{~m}$ dlameler

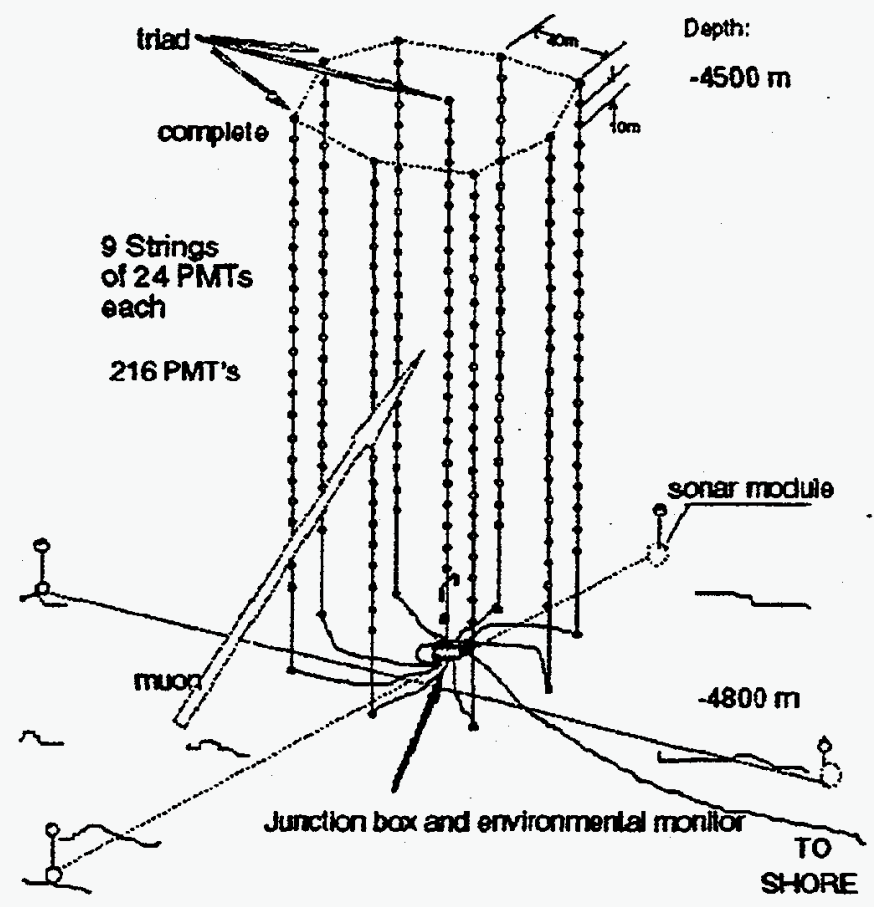

Fig. 1. The DUMAND concept for an underwater detector. connections at depth. Thus, additional strings are deployed individually from a marine research vessel with a cable winch capable of reaching depths of 5 $\mathrm{km}$. Each string is then added to the array by connecting it to the junction box. Making this connection requires either a manned submersible with robotic arms or a similarly-equipped remotely operated vehicle (ROV). An undertaking of this scope in an unusual environment (for physics experiments, at least) requires a step-wise approach. Ref. 2 details the path that DUMAND forged over the years. It included measurements of the properties of the site (environmental characteristics such as currents, optical properties, bioluminescence and radioactivity), the deployment of autonomous recoverable strings and measurements of the muon flux as a function of depth. The latter measurement [3], conducted in 1987 used a short prototype string of seven PMTs (the SPS) lowered from a research vessel to a maximum depth of $4050 \mathrm{~m}$ (Fig. 2). This was considered the proof of principle for measuring muons with strings of PMT's deployed in the ocean. In 1993 a single string, the junction box, and the cable to shore were deployed. While many aspects of the deployment operation were successful, a slow leak in one of the electrical units (the string controller) caused the string to fail and ultimately resulted in damage to the junction box as well. The string was subsequently recovered. Preparations for the deployment of the triad in Fig. 1 continued but funding for the DUMAND project ended in 1996.

Even the very short time (about 18 hours, net) the SPS was in the water and taking data (Fig. 2) enabled a competitive upper limit to be placed on high energy electromagnetic cascades induced by electron neutrinos from active galactic nuclei [4]. Fig. 3 compares the upper limits on high energy neutrino fluxes from several experiments to the predictions of theoretical models. Note the sharp dip in the limit at the Glashow 
resonance energy of $6.3 \mathrm{PeV}$. The mass of the water effectively viewed by these seven PMT's is estimated to be 2 megatons at $100 \mathrm{TeV}$ [3].

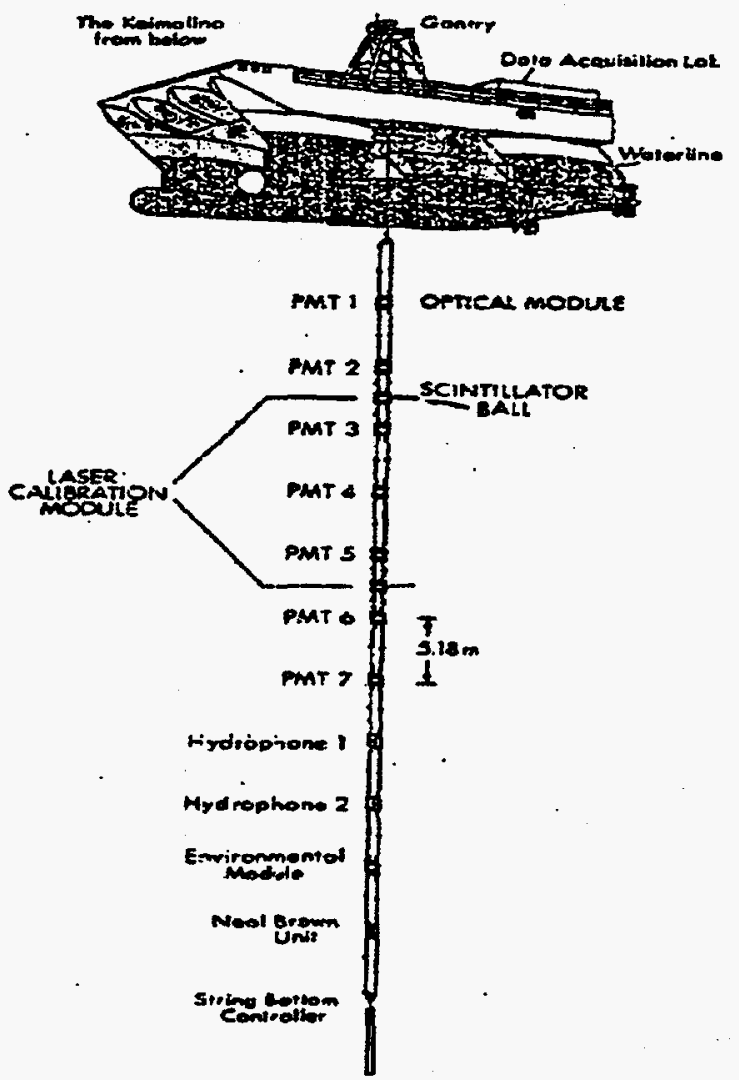

Fig. 2. The Short Protctype String [3].

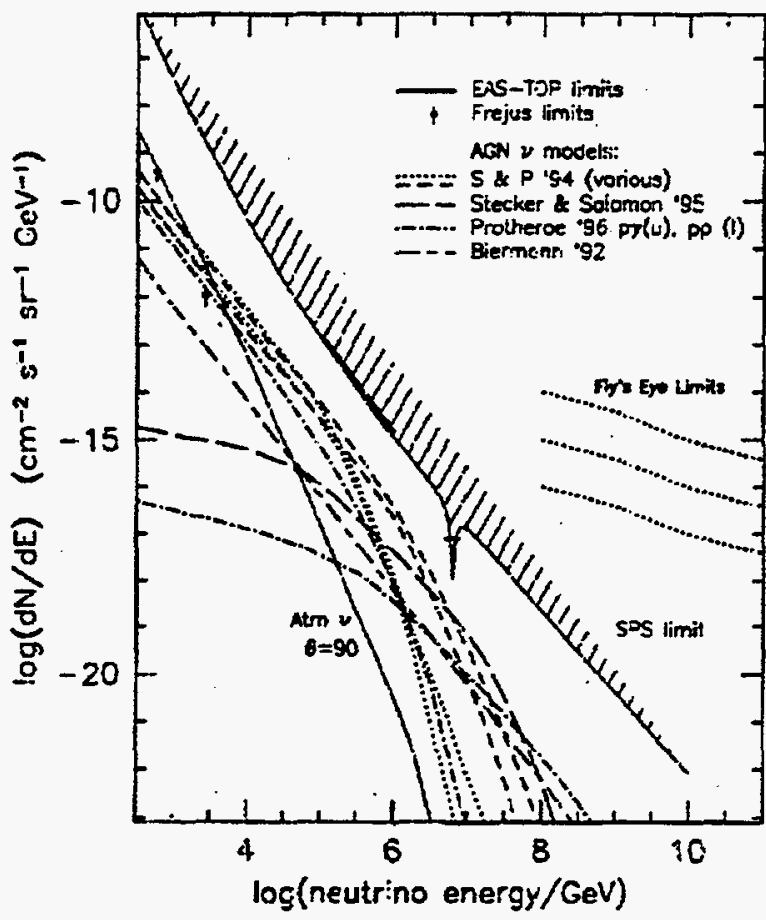

Fig. 3. Limits on neutrino fluxes set by the SPS and other experiments compared to model predictions[4].

The pioneering experience of the DUMAND project will continue to be of value for the field and for the projects that follow.

\section{NESTOR}

The NESTOR project began in 1989 with measurements conducted in the Mediterranean Sea at a site $14 \mathrm{~km}$ off the Peloponnesian coast near the town of Pylos [5]. The acronym stands for NEutrinos from Supernovae and TeV Ocean Range, but the project is really named for the wise king of Pylos who lived around $1500 \mathrm{BC}$ and whose palace overlooked the Bay of Navarino, the staging area for NESTOR's deployments. This site offers a number of advantages, in addition to being conveniently located for a European-based international collaboration. The Mediterranean generally lacks the large swells of the Pacific and has periods of great calm. The depth, $3800 \mathrm{~m}$, is quite adequate and the relatively short distance to shore reduces ship time and cable costs. The water quality is similar to that of the DUMAND site, the site floor is level with low currents, and the logistical advantages of this location including the shore infrastructure have continued to improve with time. NESTOR has an ideal site and shore facilities. 
The NESTOR concept differs substantially from DUMAND's. Each optical module (OM) is fastened to a mechanical frame that derines the position of the OM with respect to the other OM's in the six-pointed, starshaped structure (Fig. 4). (Thus, it is possible to position the OM's closer to each other and attain a lower

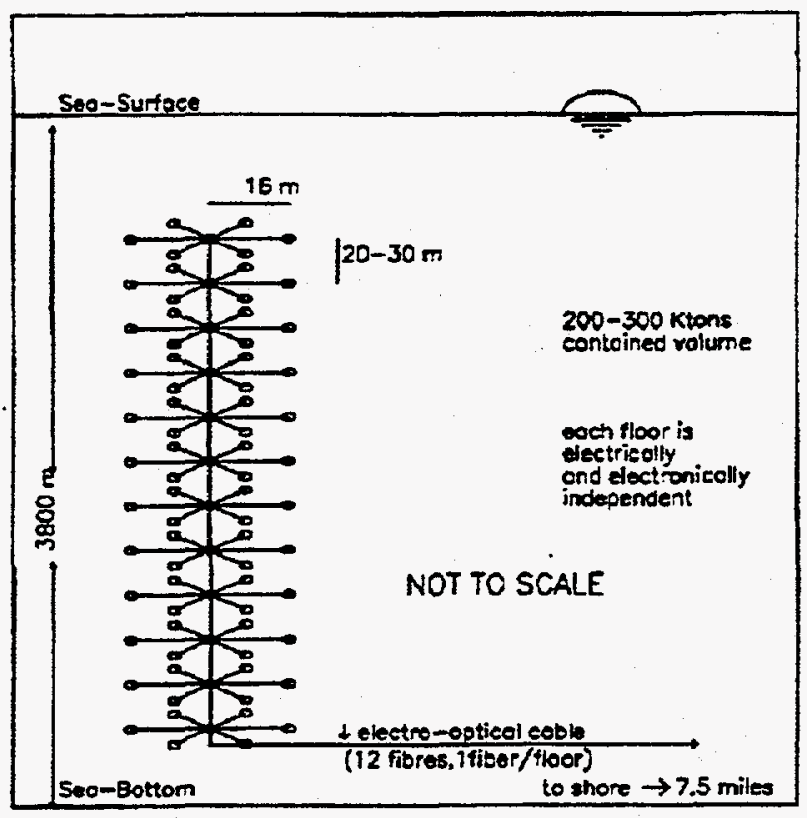

Fig. 4. The NESTOR tower concept.

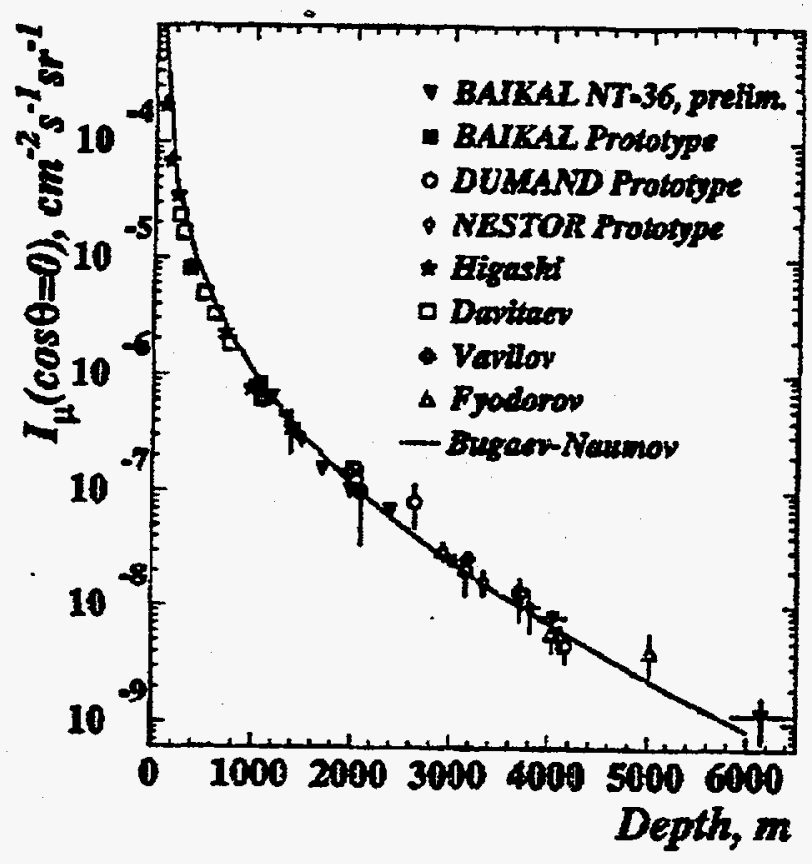

Fig. 6. Muon flux versus depth [6]. crane combination is typically less expensive to operate than the research vessels employed by DUMAND. Thus, the differences between DUMAND and NESTOR are an emphasis on a lower energy threshold, a on top of the other during a single campaign to

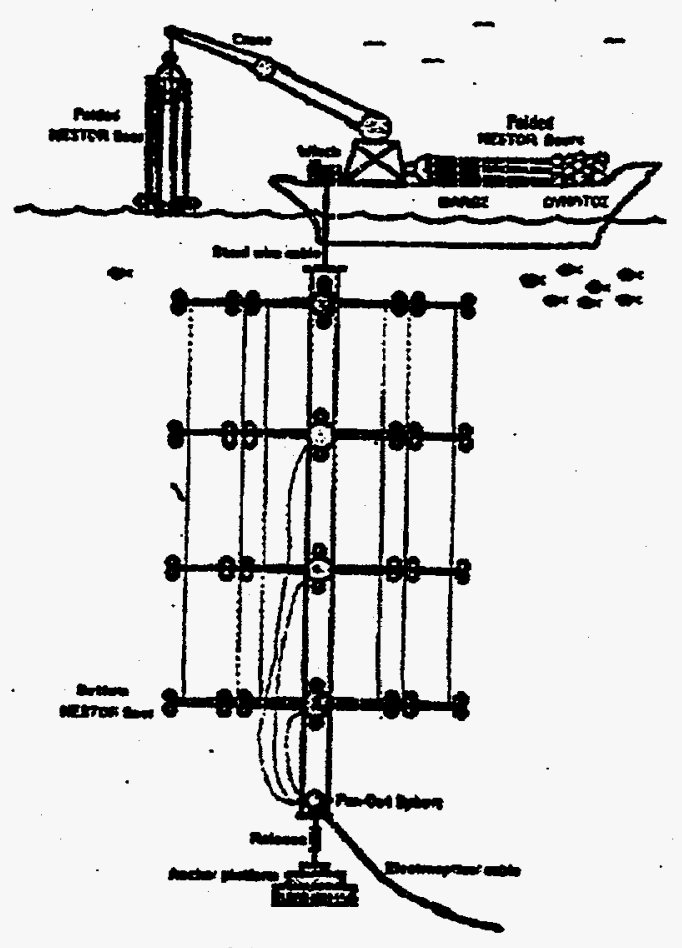

Fig. 5. Deployment of "stars" from a barge.

form a tower. But perhaps the most consequential difference is that all the electrical connections (between the stars and to the fanout sphere and e/o cable) are made on surface at or before the time of deployment rather than afterward, at depth. Manned submersibies and ROV's are thus not required. The use of a mechanical frame rather than a string also has consequences. A crane is used to lower the star into the water (Fig. 5). Such cranes, mounted on large flat barges that can also store the stars, are found relatively close to the site and, with a glassy sea, work well. This barge5

energy threshold than would be possible with strings). These structures can be deployed one 
different array topology and method of deployment. NESTOR envisions an eventual array having seven towers, each with twelve stars, capable of doing physics at both lower and higher energies. A tower could also serve as a detector for a CERN long-baseline neutrino oscillation experiment. Extrapolation to a full $\mathrm{km}$-scale detector would likely involve the use of strings at the periphery of the seven-tower array.

NESTOR has aiso measured the down-going muon flux as a function of depth. These measurements, along with the earlier measurements from DUMAND and others [6] are shown in Fig. 6. In May of 1997 a test deployment exercise was conducted in which two fully-extended stars were towed from Pylos to the site, lowered to a depth of $2600 \mathrm{~m}$, and then brought back to the surface and shore. The purpose of this test was to demonstrate the deployment and recovery technique. It would also have been possible to collect data had it not been for an electrical short in a connector. A larger scale exercise is planned for 1998 in which up to six stars will be deployed (NESTOR-A), this time connected to an electro-optical cable to shore.

\section{ANTARES}

The most recently-formed collaboration to begin working in this field is named ANTARES, which stands for Astronomy with a Neutrino Telescope and Abyss Environmental RESearch. Antares is also the name of a bright red star of the first magnitude having several hundred times the diameter of the Sun and several thousand times the Sun's luminosity. The name (of the star) comes from a Greek phrase meaning "rival of Ares" (i.e., rival of the planet Mars). ANTARES (the project) is located in the Mediterranean with a test site about $25 \mathrm{~km}$ off the coast of Toulon at a depth of $2400 \mathrm{~m}$ [7]. Their concept closely resembles that of DUMAND in that strings are used and connections will be made at depth with a manned submersible or ROV. Although ANTARES is still in an R\&D phase and plans will certainly evolve, there are likely to be some differences from DUMAND, however. Smaller phototubes $(20 \mathrm{~cm}$ or $25 \mathrm{~cm}$ diameter rather than 38 $\mathrm{cm}$ ) are envisioned, and a local fast coincidence between pairs of phototubes will be used to reduce the effective noise rate from the ${ }^{40} \mathrm{~K}$ decays. An electronic system with a greater degree of digitization and information retrieval (very similar to the one described in the next section) will be used.

ANTARES began by developing three test strings for measuring environmental properties - sedimentation rates, bio-luminescence, and optical properties of the water. One of the three autonomous, recoverable strings is shown schematically in Fig. 7. With this device the background counting rates due to ${ }^{40} \mathrm{~K}$ radioactivity and bio-luminescence are measured. After a period time on the ocean floor, which may be up to a month or longer, the acoustic release at the base of a string is triggered, the string rises to the surface and is located by means of the beacon at the top. The data stored in the instrumentation vessel are then recovered. The site they are now using is well-suited for R\&D because marine research organizations (IFREMER) have bases near Toulon and ocean floor there has been studied. However, the strings that are tested here will be used to investigate deeper and clearer sites in the Mediterranean that would be more suitable for the next size of detector $\left(0.1 \mathrm{~km}^{2}\right)$. 
The Toulon site will be used to develop all aspects needed to plan a $0.1 \mathrm{~km}^{2}$ detector, including making connections at depth. The culmination of this initial R\&D stage is scheduled for late 1999 and is the

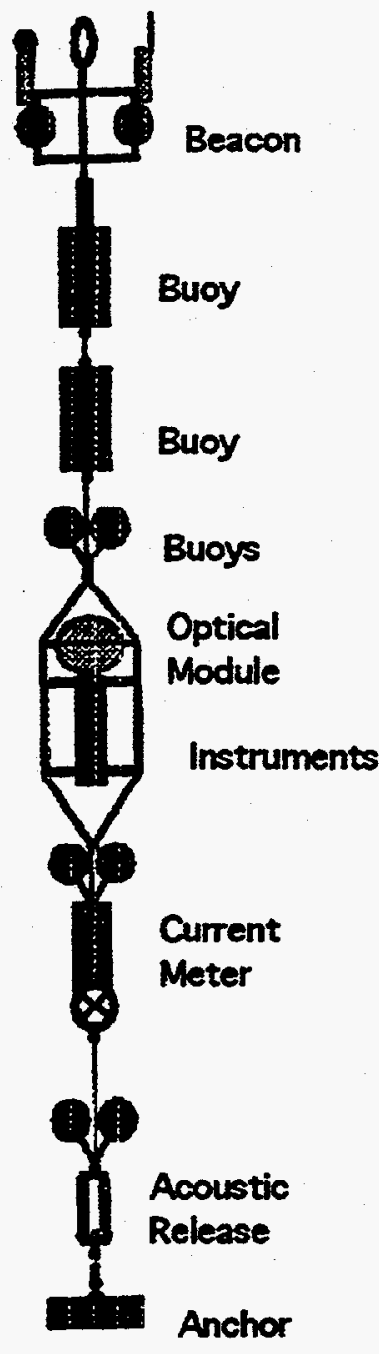

Fig. 7 ANTARES string for measuring $40_{\mathrm{K}}$. "demonstrator" - a three string array. This is shown schematically in Fig. 8. Each of the three strings consists of 16 pairs of OM's, with each pair of adjacent PMT's operated in local coincidence. The spacing of the strings would be about $100 \mathrm{~m}$.

Based on the experience with deploying and connecting strings gained with the three string array, ANTARES will propose a larger array, to be located at a permanent site to be chosen also in the next two years. This would be the stage intermediate between the three string array and a full $\mathrm{km}$-scale detector.

\section{LBNL}

One of the central problems in neutrino detection is to recognize relatively rare signals in a background larger by many orders of magnitude. The number of downward going muons that are mis-reconstructed as upward going muons, i.e., as neutrino events, must be small compared to the signal. Depending on the depth of the detector the ratio of downward to upward going muons can be in the range (roughly) of $10^{6}$ (at $1200 \mathrm{~m}$ ) to $10^{4}$ (at $4000 \mathrm{~m}$ ). The events themselves, regardless of muon direction, can be quite complex muons may come in bundles rather than singly, above $\sim 500 \mathrm{GeV}$ secondary electromagnetic showers will 
become more frequent along the track, and electron neutrinos will induce relatively short but very bright electromagnetic cascades. Scattering of the Cherenkov light in the medium will disperse the arrival of photons that would otherwise be simultaneous. These complex events are reflected in a complex output signal at the anode of the photomultiplier tube - complex in form and large in dynamic range. When the events of interest are rare it is important that the maximum possible information from an event be recorded and transmitted to the shore station. The long distance to shore requires that this information be digitally encoded. The complexity of the event argues for digitally encoding the entire wave form at the output of the PMT (rather than just time of arrival and total charge.) Lawrence Berkeley National Laboratory is developing a digital system, suitable for a $\mathrm{km}$-scale array, that addresses this challenge [8]. The main element in this system is the Digital Optical Module (DOM), and the key element in the DOM is an application specific integrated circuit called the Analog Transient Waveform Recorder (ATWR) developed by $S$. Kleinfelder at LBNL.

The ATWR is a series of switched capacitors, which are connected and disconnected from the input signal in a time sequence that results in a capture of the transient signal in time buckets, the width of which can be as small as a nanosecond. A 128 channel device would thus provide a time resolution of 1 nanosecond for a period of 128 nanoseconds, sufficient in principle to resolve photons from parallel muons a few meters apart. Incorporating several of these rows of switched capacitors into a single chip makes it possible to record dynode signals as well, and also a clock signal to provide timing information. Other features are a very low power consumption and an adjustable width for the time bucket.

In collaboration with LBNL, two prototype DOM's were built by the Jet Propulsion Laboratory and deployed at a depth of about $1500 \mathrm{~m}$ in Antarctic ice on one of the strings comprising the AMANDA-B array. This afforded an opportunity to test the performance of the DOM's triggered by an operating array of phototubes. Fig. 9 shows a typical waveform taken with one of the two DOM's. The discriminator thresholds on each PMT were high, at a level of about five photoelectrons. (This was not intended.) Also, the thresholds on the individual AMANDA OM's were higher than usual during this particular data-taking period. The result is that both the DOM's and the AMANDA array as a whole were biased toward energetic events producing a lot of light in the whole array and close to the DOM's. This, however, is ideal for observing complex events. Such an event is illustrated in Fig. 10. After a very large initial burst of photons (which itself has a structure), there are three smaller peaks, having one or two photoelectrons each. A preliminary analysis of the distribution of peaks in height and time (for many such waveforms) suggests that these structures arise mainly from sources of light separated in space and in time, and are not solely the consequence of scattering-induced delay. When the coincidence data from the rest of the AMANDA array become available in late 1997, it will be possible to determine the tracks of the muons that produced these complex events. In any case, this is the first observation of complex waveforms in a deep neutrino telescope, and it suggests that it is indeed important to capture this information. 
The digital optical modules are the main part of an overall system for controlling the array from shore and the transmitting of the data back to shore. Other components of the network will include local string modules (that serve eight DOM's), string controllers, and network nodes. The network must also

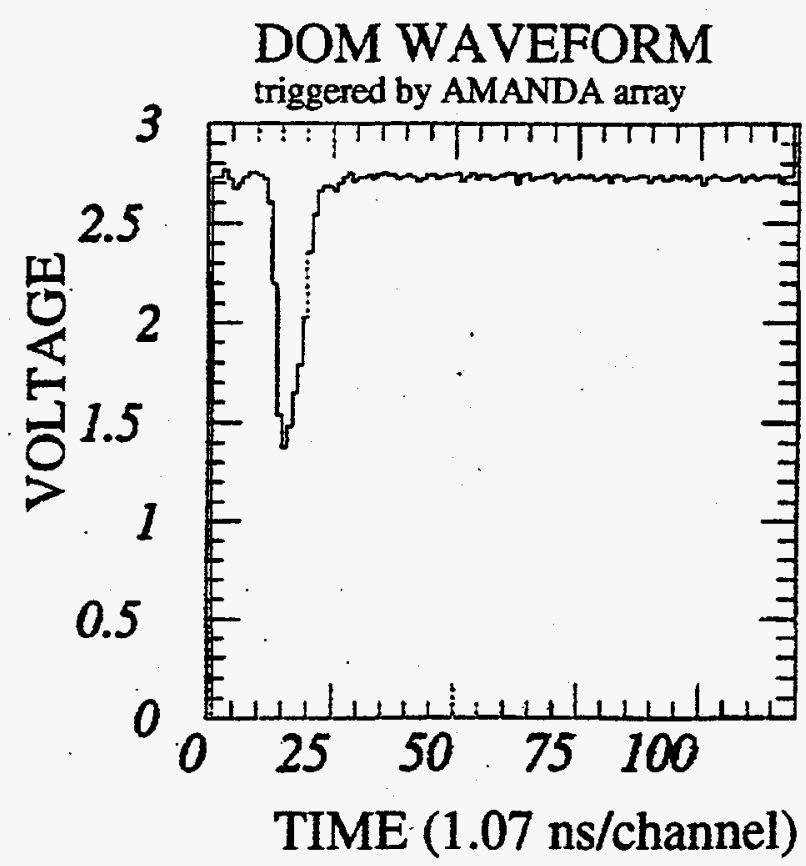

Fig. 9. Digital waveform obtained with the LBNLJJPL DOM. The single peak contains about 6 photoelectrons.
DOM WAVEFORM triggered by AMANDA array

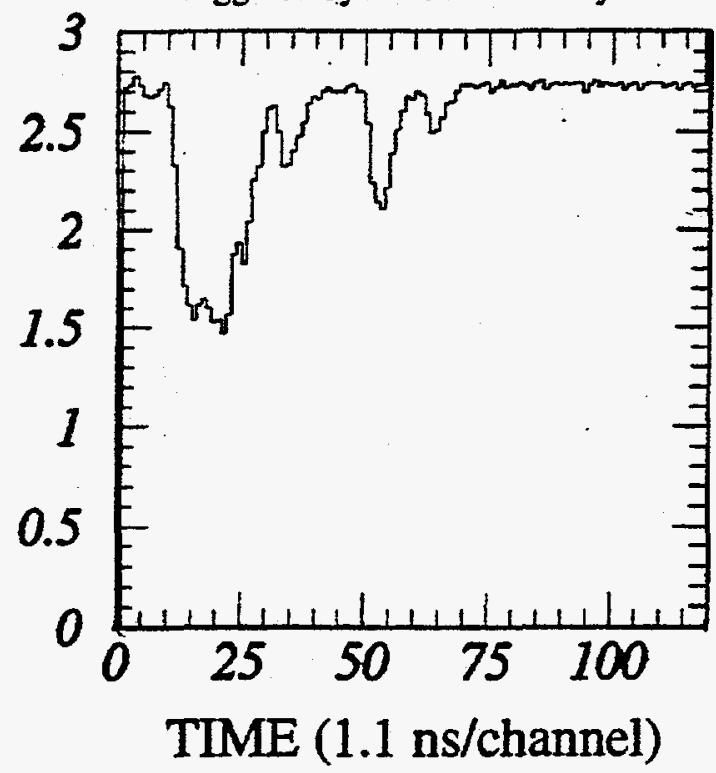

Fig. 10. Digital waveform obtained with the LBNL/JPL DOM. The small peak at the right is a single photoelectron.

provide a timing capability, that is, a calibration or 'time-stamping' of the local slow-speed (15 MHz) clock in each DOM. Design and prototyping of this system is in progress.

\section{Outlook}

Progress towards a km-scale neutrino detector necessarily involves stages. Both NESTOR and ANTARES are set in the near future to pass the critical milestone - a working prototype array that is able to identify upgoing muons. Based on their progress to date, the outlook for both projects is certainly bright.

Although the ocean, in general, is not an easy or forgiving place to work, it offers a number of advantages. The optical properties are good; in particular, the scattering length in ocean water is about twice that of polar ice (at the location of AMANDA-B). There are a number of possible sites offering depth, proximity to shore and the requisite infrastructure. Greater depth translates to reduced background and fewer misreconstructed events. The sites at middle latitude cover a larger portion of the sky than can be viewed from the Pole. Because equipment is recoverable from the ocean, it is also possible to maintain it. And finally, the large amount of oceanographic research as well as the commercial marine technology enable synergistic collaborations. An example of this is LAERTES, a NESTOR-affiliated organization that will promote interdisciplinary research using the NESTOR tower and shore connection. ANTARES also has strong connections with the oceanographic community via IFREMER. 
I would like to conclude with a brief perspective on the field of high energy neutrino astronomy. It is informative to compare it with the more mature field of low energy, solar neutrino astronomy.

$\begin{array}{lll}\text { Stage } & \text { Low Energy } & \text { High Energy } \\ \text { First Ideas } & 1945 \text { (Pontecorvo) } & 1960 \text { (Markov) } \\ \text { Serious Discussion } & \text { early 60's } & \text { early 70's } \\ \text { Proposals } & 1964 & 1978 \\ \text { First Detection of v's } & \text { early '70s (Homestake) } & \text { mid 90's (Baikal) }\end{array}$

From this terse comparison it is evident that H.E. neutrino astronomy is presently in about the same stage as L.E. neutrino astronomy was twenty to twenty-five years ago. If the comparison holds, and I think it does, we can expect a rapid growth in this new field and also a long and scientifically rich future.

\section{Acknowledgments}

I would like to thank L. Resvanis and colleagues, and J.J. Aubert and colleagues, for introducing me to NESTOR and ANTARES. I hope I have done justice to their efforts. I am pleased to acknowledge my colleagues at LBNL - D. Nygren, who has led the development of the digital system - and W. Chinowsky, H. Crawford, and G. Smoot. At JPL, J. Ling, S. Jackson and their colleagues built the DOM prototypes that delivered data. D. Lowder of U.C. Berkeley and G. Przyblyski of LBNL brought the DOM's to the Pole. Thanks to the AMANDA collaboration for their support in deploying them.

\section{References}

[1] T.K. Gaisser, F. Halzen, and T. Stanev, Phys. Rep. 258 (1995) 708.

[2] A. Roberts, Rev. Mod. Phys. 64 (1992) 259.

[3] J. Babson, et al., Phys. Rev. D42 (1990) 3613

[4] J.W. Bolesta, et al., Proc. Int. Cosmic Ray Conf., Durban, 1997, ed. M.S. Potgieter, et al., HE 4.1.11, Vol. 7, p. 29.

[5] L.K. Resvanis, Proc. 2nd NESTOR Int. Workshop, Pylos, Greece (1992), p. 15; L.K Resvanis, Proc. U.S Mtg. on Future Prospects for $\mathrm{Km}^{3}$-Scale Neutrino Detectors, Arcadia, CA, (1996) p. 43.

[6] I.A. Belolaptikov, et al., Proc. Int. Cosmic Ray Conf., Rome (1995). Fig. 6 is from ref. 7.

[7] F. Blanc, et al., ANTARES Proposal, May 1997, DAPNLA-97-03; V. Bertin, in Recontre de Moriond (1997).

[8] D. Nygren, et. al., LBL-38321, Proposal to US DOE, Feb. 1997; H. Kirkahm, et. al, to be published. 